\title{
Steroid and Neuronal Regulation of Ecdysone Receptor Expression during Metamorphosis of Muscle in the Moth, Manduca sexta
}

\author{
Carol D. Hegstrom, ${ }^{1}$ Lynn M. Riddiford, ${ }^{2}$ and James W. Truman ${ }^{2}$ \\ ${ }^{1}$ Department of Psychology, University of California, Berkeley, California 94720-1650, and '2Department of Zoology, \\ University of Washington, Seattle, Washington 98195-1800
}

\begin{abstract}
Ecdysteroids regulate the remodeling of the dorsal external oblique 1 (DEO1) muscle during metamorphosis in Manduca sexta (Hegstrom and Truman, 1996a). We show that the temporal and spatial patterning of the $A$ and $B 1$ isoforms of the ecdysone receptor (EcR) within muscle DEO1 corresponds with the developmental fates of the fibers. Using antibodies directed to specific isoforms of EcR, we show that the expression of various EcR isoforms in myonuclei differ among the five fibers of DEO1 and correspond with the developmental response of the muscle to the changing steroid titers and to the pattern of innervation. Muscle degeneration and apoptosis of myonuclei in all fibers are correlated with the expression of only EcR-A just before pupal ecdysis and then with the expression of low levels
\end{abstract}

of both EcR-A and EcR-B1 shortly after pupation. Only the first fiber of muscle DEO1 participates in the regrowth of the adult muscle, and only this fiber shows an upregulation of EcR-B1 that is evident at $3 \mathrm{~d}$ after pupal ecdysis. Denervation of the muscle prevents both the upregulation of EcR-B1 and myoblast proliferation. We conclude that the developmental fate of muscle DEO1 during metamorphosis is orchestrated by interactions between rising and falling ecdysteroid titers, the pattern of expression of EcR isoforms by the muscle, and interactions with other cells in the local environment.

Key words: muscle; nuclear hormone receptor; ecdysteroid; metamorphosis; receptor isoform; steroid receptor
Developmental hormones, such as steroid and thyroid hormones, act as global chemical messengers to coordinate developmental events. An intriguing feature of their action is that different cells exposed to identical hormonal signals may nevertheless undergo markedly different cellular responses. One possible basis for this variability in response is differences in the types of hormone receptors that the cells possess. The receptors for these hormones are ligand-regulated transcription factors that are members of the nuclear receptor superfamily. They are characterized by a variable N-terminal region, a highly conserved DNA-binding domain with two zinc fingers, and a ligand-binding domain that is much less well conserved (Evans, 1988; Beato et al., 1995).

There are often multiple forms (isoforms) of receptors for a given hormone. For example, in insects the gene for the ecdysone receptor (EcR), which was first isolated from Drosophila (Koelle et al., 1991), encodes multiple isoforms that share common DNAand ligand-binding domains but differ in the $\mathrm{N}$ terminus (Talbot et al., 1993). In Drosophila, there are at least three isoforms: EcR-A, EcR-B1, and EcR-B2. In the moth, Manduca sexta, there are two distinct isoforms that are likely homologs of EcR-B1 (Fujiwara et al., 1995) and EcR-A (Jindra et al., 1996).

In many systems the temporal and spatial patterns of expression of the various receptor isoforms correlate with different types of hormonal responses (Wills et al., 1991; Talbot et al., 1993). This is especially evident for EcR (Talbot et al., 1993; Truman et al., 1994). For example, in the CNS of Drosophila, high levels of

Received Aug. 19, 1997; revised Dec. 12, 1997; accepted Dec. 17, 1997.

This study was supported by Grants from National Institutes of Health Grants NS 13079 to J.W.T. and NS 29971 to J.W.T. and L.M.R. and National Science Foundation Grant IBN 9514187 to L.M.R. We thank Ta Deng, Crystal Garnes, and David Champlin for preparation and testing of the Manduca EcR antibodies.

Correspondence should be addressed to Carol Hegstrom, Department of Psychology, 3210 Tolman Hall, University of California, Berkeley, CA 94720-1650.

Copyright (C) 1998 Society for Neuroscience $0270-6474 / 98 / 181786-09 \$ 05.00 / 0$
EcR-A are correlated with programmed cell death (Robinow et al., 1993), whereas expression of EcR-B1 is correlated with proliferation or remodeling (Truman et al., 1994).

The expression of a receptor isoform may be regulated by its ligand, as has been shown for glucocorticoids (Antakly et al., 1990; Miller et al., 1993; Schmidt and Meyer, 1994) and for thyroid hormone (Yaoita and Brown, 1990; Kawahara et al., 1991; Wills et al., 1991). Alternatively, the expression of a receptor may be determined by interactions among cells within their immediate local environment (Hughes et al., 1985; Hughes and Krieg, 1986; Lubischer and Arnold, 1995; Glauser and Barakat, 1997).

In the moth, $M$. sexta, metamorphosis causes a profound remodeling of the neuromuscular system. Larval motoneurons persist through this transformation, but their larval muscle targets degenerate and are replaced by new muscles that grow during adult differentiation (Nüesch, 1985). Studies on the musculature of the legs (Consoulas et al., 1996; Consoulas and Levine, 1997) and of the abdominal body wall (Truman and Reiss, 1995; Hegstrom and Truman, 1996a,b) show that muscle remodeling is dependent on steroid hormones, the ecdysteroids, and a dialogue between motoneuron and muscle. The combination of influences is especially evident during adult muscle growth when proliferation of myoblasts depends on both ecdysteroids and innervation (Hegstrom and Truman, 1996a; Consoulas and Levine, 1997). We show in this study that innervation regulates the choice of EcR isoforms expressed in growing muscle. This choice may then determine the nature of the response of the muscle to changing steroid titers.

\section{MATERIALS AND METHODS}

Animals. Larvae and pupae of the tobacco hornworm (M. sexta) were reared individually on an artificial diet (modified from Bell and Joachim, $1976)$ at $26^{\circ} \mathrm{C}$ under a long-day (17/7 hr light/dark) photoperiod. In some instances, animals were raised under short-day conditions $(12 / 12 \mathrm{hr}$ 
light/dark) to produce pupae that subsequently went into diapause, a state of developmental arrest. Animals were staged relative to wandering (day W0) or to pupal ecdysis (day P0). Both males and females were used for experiments.

Steroid manipulations. The prothoracic glands are the primary source of ecdysteroids in Manduca. To remove this endogenous source of steroids, animals were ligated between the thorax and abdomen with a hemostat, and the thorax and head were severed and discarded. Although this procedure precipitates a decline in ecdysteroid titer (Weeks et al., 1992), the isolated abdomens survive for weeks. Animals were ligated within 3 hr after pupal ecdysis.

In some cases, isolated abdomens were given injections of the ecdysteroid 20-hydroxyecdysone (20E; Sigma, St. Louis, MO). 20E was dissolved in saline (Ephrussi and Beadle, 1936) at $1 \mathrm{mg} / \mathrm{ml}$. To mimic the early rise in the adult peak of ecdysteroids, we isolated abdomens a few hours after pupal ecdysis and then injected $10 \mu \mathrm{g}$ of $20 \mathrm{E}$ into the abdomens $1 \mathrm{~d}$ later. To break diapause and initiate adult development in diapausing pupae (Bradfield and Denlinger, 1980), we injected $20 \mu \mathrm{g}$ of $20 \mathrm{E}$ into the dorsal thorax of pupae that had been immobilized by chilling. The site of injection was covered with a drop of melted dental wax to prevent bleeding. Injected preparations were maintained at $26^{\circ} \mathrm{C}$ for $24-72 \mathrm{hr}$.

Injections of bromodeoxyuridine. A saturated solution of bromodeoxyuridine (BrdU; Sigma) was prepared in distilled water $(\sim 30 \mathrm{mg} / \mathrm{ml}$ at $37^{\circ} \mathrm{C}$ ). Fifty microliters of this solution were injected into the dorsal thorax of pupae of various stages. The site of injection was then covered with melted wax to prevent bleeding. The animals were maintained at $26^{\circ} \mathrm{C}$ for $6-72 \mathrm{hr}$. The animals were then prepared for immunohistochemistry as described below.

Immunocytochemistry. For all histochemical reactions, the dorsal fourth abdominal segment was dissected free in normal saline (Ephrussi and Beadle, 1936) and fixed for $1 \mathrm{hr}$ at room temperature with $4 \%$ paraformaldehyde in $0.1 \mathrm{M}$ PBS, $\mathrm{pH}$ 7.2. The tissue was rinsed in PBS with $0.3 \%$ Triton X-100 (PBS-TX). For pupae that had been injected with BrdU, the tissue was then incubated for $1 \mathrm{hr}$ in $2 \mathrm{~N} \mathrm{HCl}$ to denature the DNA.

The fixed or the fixed and acid-treated tissue was rinsed several times in PBS-TX and then blocked in $10 \%$ normal goat serum in PBS-TX from $2 \mathrm{hr}$ (room temperature) to overnight $\left(4^{\circ} \mathrm{C}\right)$. The tissue was then incubated with the primary antibody diluted in PBS-TX with $1 \%$ normal serum and $0.05 \%$ sodium azide. Mouse monoclonal antibodies to Manduca EcR-B1 (Jindra et al., 1996) and to BrdU (Becton Dickinson, Mountain View, CA) were diluted 1:1000 and 1:200, respectively. The rabbit polyclonal antibody specific to EcR-A (D. Champlin and L. M. Riddiford, unpublished observations) was diluted 1:1000. After incubation for $24-72 \mathrm{hr}$ at $4^{\circ} \mathrm{C}$, the tissue was rinsed several times in PBS-TX and then exposed to peroxidase-conjugated goat anti-mouse IgG or donkey anti-rabbit IgG (Jackson ImmunoResearch, West Grove, PA; 1:1000 in PBS-TX with 1\% normal serum) for $24-48 \mathrm{hr}$, after which the tissue was again rinsed several times. The labeling was revealed using diaminobenzidine (Sigma) as a substrate, according to the method of Watson and Burrows (1981) with the addition of $4.3 \times 10^{-6} \mathrm{M} \mathrm{NiCl}$ to intensify the staining. The tissue was then rinsed, dehydrated in an ethanol series, cleared in xylene, and mounted in Permount mounting medium.

The nuclei were counted in the following manner. The muscle was viewed at $20 \times$, and the stained nuclei of the central region of fiber 1 were drawn using a camera lucida attachment. A square equivalent to 10,000 $\mu \mathrm{m}^{2}$ was placed randomly within the central region of fiber 1 , and the labeled nuclei that lay within the boundaries of the square were counted. The lengths of all the stained nuclei within the sampled area were measured and converted into micrometers. Although the intensity of staining differed among nuclei at different stages and fibers, no attempt was made to quantify staining intensity.

Confocal Microscopy. A laser scanning confocal microscope (Bio-Rad 600; Bio-Rad, Hercules, CA) was used for observation of double-labeled tissues. The tissue was prepared for immunocytochemistry as described above, with the exception that the secondary antibody was fluorescently labeled. After the second label was applied, the tissue was mounted on coverslips, dehydrated through a graded ethanol series, cleared in xylene, and mounted in DPX (Fluka BioChemika, Ronkonkoma, NY) mounting medium.

The COMOS software for the confocal microscope was used to obtain histograms of intensity of staining of nuclei. An area corresponding to an individual nucleus was selected, and the average pixel intensity was measured.

Double labeling with terminal deoxynucleotidyl transferase-mediated dUTP-biotin nick end labeling and EcR-B1. The terminal deoxynucleotidyl transferase-mediated dUTP-biotin nick end-labeling (TUNEL) method of labeling free ends of DNA was used to assay for apoptotic nuclei as described by Gavrielli et al. (1992). Muscle fibers were fixed in $4 \%$ paraformaldehyde for $1 \mathrm{hr}$ and then rinsed in several washes of PBS-TX. The tissue was blocked and stained for EcR-B1 as described above and then rinsed with several washes of PBS-TX before incubation with a fluorescently labeled secondary antibody (BODIPY-Fl goat anti-mouse secondary antibody; Molecular Probes, Eugene, OR) for $24 \mathrm{hr}$. The tissue was then rinsed several times, post-fixed for $30 \mathrm{~min}$ in $4 \%$ paraformaldehyde at room temperature, and then rinsed again. The first fiber of dorsal external oblique 1 (DEO1) muscle was dissected free and placed in TdT buffer (Life Technologies, Gaithersburg, MD) for $\sim 10$ min. The TdT buffer was then replaced with fresh TdT buffer containing $100 \mu \mathrm{M}$ dUTP/biotinylated dUTP (Boehringer Mannheim, Indianapolis, IN) $\operatorname{mix}(2: 1)$ and $0.3 \mathrm{U} / \mu \mathrm{l}$ terminal deoxynucleotidyl transferase (Life Technologies). The fibers were incubated in this solution for 3-4 hr at $37^{\circ} \mathrm{C}$, rinsed in several washes of PBS-TX, and incubated with avidinTexas Red (Vector Laboratories, Burlingame, CA) at a dilution of 1:500 overnight. After a final rinse, the tissue was mounted on coverslips, dehydrated in a graded ethanol series, cleared in xylene, and mounted in DPX mounting medium.

Nerve Transection. Animals of various stages (diapause, $\mathrm{P} 0$, or $\mathrm{P}+3 \mathrm{~d}$ ) were selected and anesthetized by chilling on ice. A small piece of cuticle near one spiracle of the fourth abdominal segment was removed. The nerve branch that contains the axon of motoneuron 12 (the motoneuron that innervates muscle DEO1; Levine and Truman, 1985) was visually located and transected with forceps. A small crystal of phenylthiolurea was placed in the wound area to prevent blood blackening, and the cuticle was repositioned. The wound was sealed with wax, and the animals were maintained at $26^{\circ} \mathrm{C}$ to continue development for $24-96 \mathrm{hr}$.

\section{RESULTS}

\section{Expression of EcR in muscle DEO1}

In Manduca, ecdysteroids regulate the remodeling of the abdominal body wall muscle DEO1 and its motoneuron, MN-12 (Fig. 1) (Truman and Reiss, 1995; Hegstrom and Truman, 1996a,b). The larval muscle is composed of five fibers, and all degenerate in response to the decline in the prepupal peak of ecdysteroids. The adult muscle then begins to grow from the remains of the first fiber (fiber 1) starting about day $\mathrm{P}+3$ as the ecdysteroid titer rises to form the adult peak of ecdysteroids and to cause adult differentiation. The persistence of innervation on fiber 1 is essential for the steroid-driven growth of the muscle. The remains of the more distal fibers lack innervation and degenerate in response to the same steroid signal that causes fiber 1 to regrow.

The distribution of EcR in muscle DEO1 was determined using the monoclonal antibodies (mAbs) 6A7 and 6B7. These detect an epitope that is unique to the N-terminal region of the EcR-B1 isoform, mAb 1B5, that is directed toward both an epitope in the common region included in all known Manduca EcR isoforms (Jindra et al., 1996), and a polyclonal antiserum that was raised against a fusion protein that included the peptide encoded by the A-specific exons of EcR (Jindra et al., 1996). Specificity of the antibodies in staining the muscle was determined by preincubation of the EcR-B1 and EcR-A antibodies with the fusion proteins before exposure to the tissue. The EcR-B1 fusion protein completely eliminated staining by the EcR-B1 mAb but had no effect on the EcR-A staining. Pretreatment with the EcR-A fusion protein had no effect on EcR-B1 staining and reduced, but did not eliminate, staining with the EcR-A antiserum. Despite not binding to antigen in solution, we think the residual staining is also attributable to EcR-A. One reason is that in control tissues the staining pattern detected by the EcR common region antibodies is a combination of the EcR-A and EcR-B1 staining patterns. Also, in 


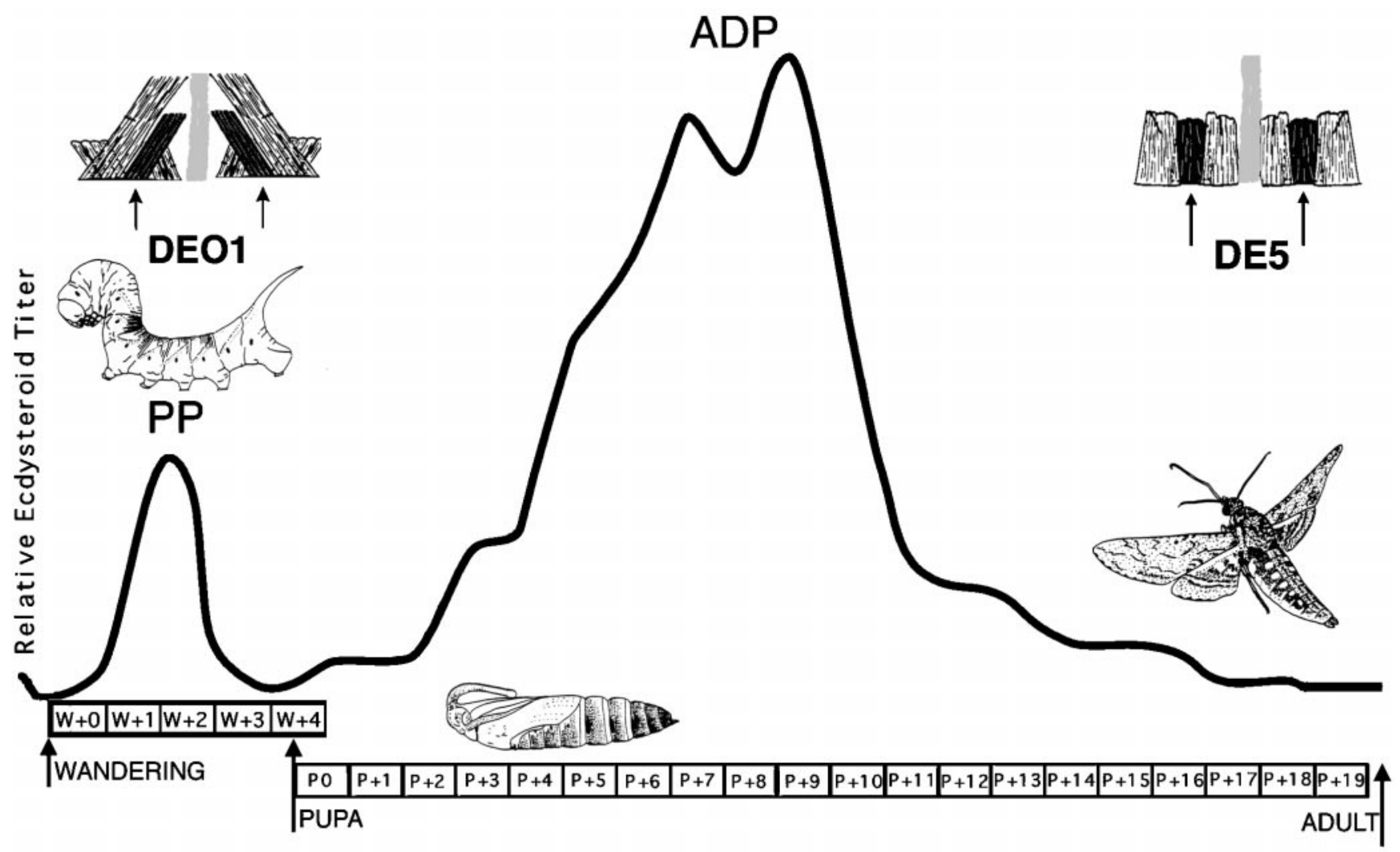

Figure 1. Relative ecdysteroid titers during the larval-pupal-adult transition. Insets, Dorsal view of an abdominal segment showing the paired external muscles that are situated on either side of the heart. The DEO1 muscles of the larva degenerate and regrow as the dorsal external 5 (DE5) muscles of the adult. $P P$, Prepupal peak; $A D P$, adult peak. Boxes represent days after wandering or after pupal ecdysis. Ecdysteroid titer data from Bollenbacher et al. (1981).

A

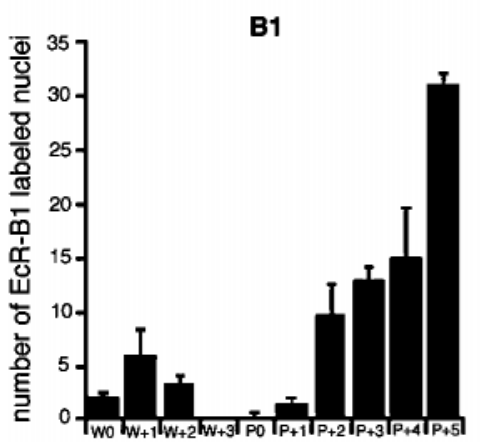

day
B

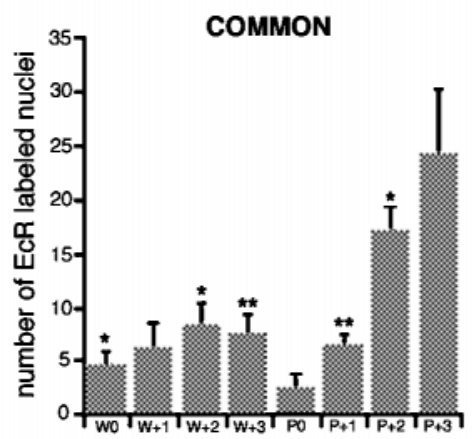

day
C

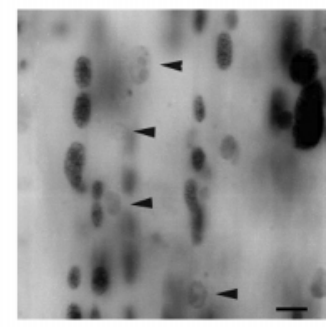

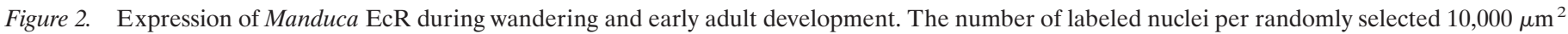

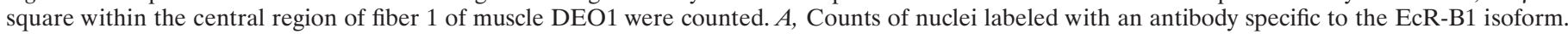

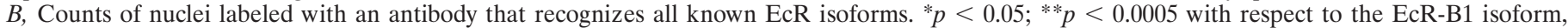

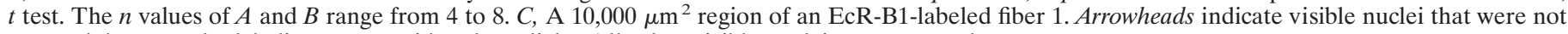
counted, because the labeling was considered too light. All other visible nuclei were counted.

Western blots, the EcR-A antiserum recognizes a band that is recognized by EcR common mAbs but not by EcR-B1 mAbs (Q. Song and L. I. Gilbert, unpublished observations).

Early in metamorphosis, EcR-B1 appeared in nuclei of all fibers of muscle DEO1 in two waves, the first after wandering and the second during early adult development (as can be seen for staining of fiber 1; Fig. 2). At the onset of wandering, few nuclei expressed EcR-B1, and this expression was at low levels. The staining levels and the number of nuclei expressing EcR-B1 increased coincident with the rise in the prepupal peak of ecdysteroids at day $\mathrm{W}+1$. By day $\mathrm{W}+3$, when the prepupal ecdysteroid titer had decreased again (Fig. 1), EcR-B1 was undetectable (Fig. 2). On day P0, just after pupal ecdysis, the ecdysteroid titer was low, and EcR-B1 was also very low or absent. Later, as adult development commenced and the ecdysteroid titer rose, the number of stained nuclei and the intensity of EcR-B1 


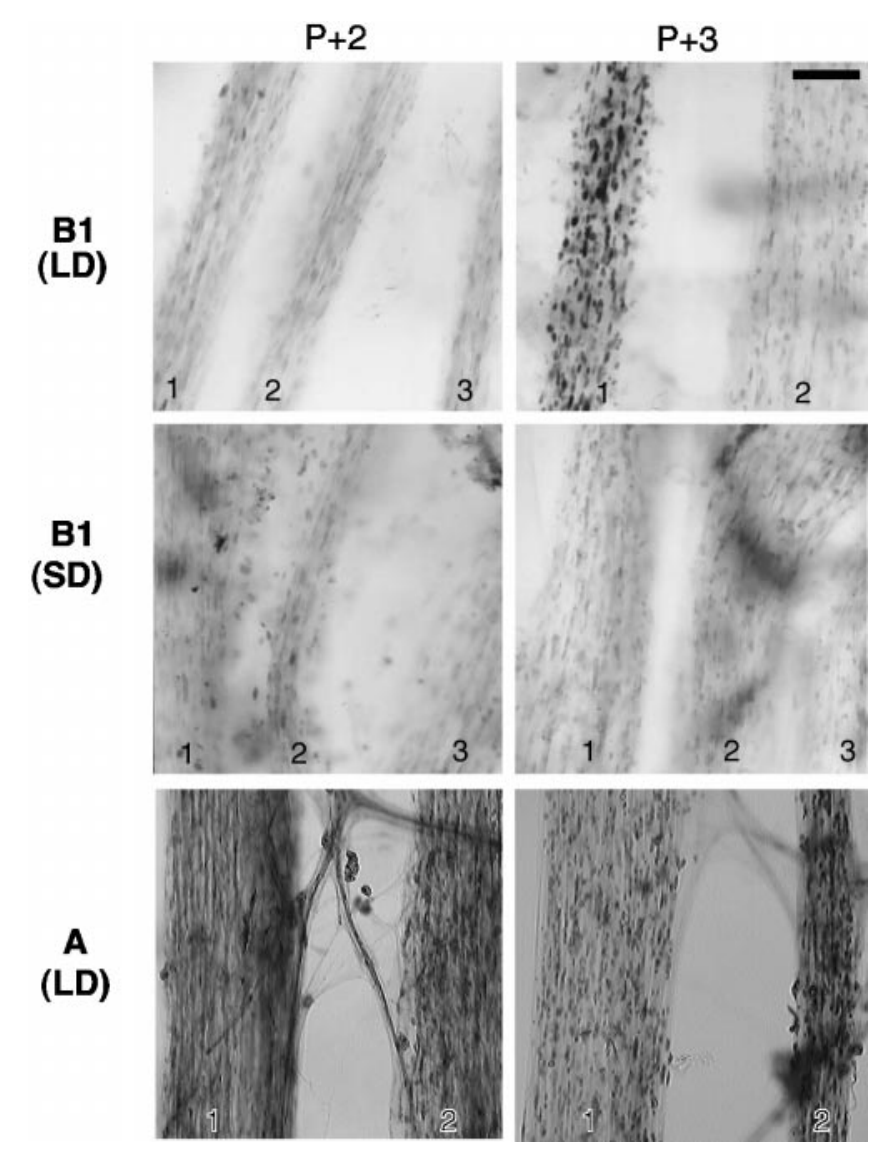

Figure 3. EcR expression in muscle DEO1 at days $\mathrm{P}+2$ and $\mathrm{P}+3 . L D$, Long-day-reared animals that will commence adult development on day $\mathrm{P}$ $+3 \mathrm{~d} ; S D$, short-day-reared pupae that arrest development on $\mathrm{P}+3 \mathrm{~d} ; B 1$, expression of EcR-B1 as indicated by immunostaining with the mAb specific to the EcR-B1 isoform; $A$, expression of EcR-A. Numbers indicate fiber number; fiber 1 is to the left. Scale bar, $100 \mu \mathrm{m}$.

staining increased and were evident through the last day that we examined, day $\mathrm{P}+5$. During wandering and through day $\mathrm{P}+2$, the EcR-B1 immunoreactivity seemed equal in nuclei of all five fibers of muscle DEO1 (data not shown). By day $\mathrm{P}+3$, however, the staining in the more distal fibers weakened, and few nuclei were labeled, but in the central region of fiber 1 the number of stained nuclei increased, and the staining intensity became stronger (Fig. 3; see Fig. 8, top). This disparity became more extreme as adult development progressed.

The pattern of staining using the $\mathrm{mAb}$ directed to a region common to all isoforms showed basically the same pattern as seen with mAbs against EcR-B1. There was, though, a prominent difference on day $\mathrm{W}+3(n=6)$, when staining for the EcR-B1 isoform was virtually absent $(n=8)$, but the common mAb showed strong staining (Fig. 2) in many nuclei. There were also slight differences in staining levels at days $\mathrm{P} 0$ and $\mathrm{P}+1$, when numbers of EcR-B1-labeled nuclei remained low. The use of the EcR-A-specific antibody confirmed that the staining seen with the common EcR mAb was, indeed, attributable to EcR-A. Staining intensity and number of EcR-A-labeled nuclei decreased at pupal ecdysis and returned to moderate levels by days $\mathrm{P}+2$ and $\mathrm{P}+3$ when, in contrast to EcR-B1, the staining was evenly distributed over all five fibers of muscle DEO1 (Fig. 3). In a few cases (12\%), EcR-A staining appeared slightly higher on fiber 1 .

\section{Regulation of EcR expression by ecdysteroids}

After pupal ecdysis, both animals destined for diapause (short-dayreared) and those that show continuous development (long-dayreared) have low but detectable levels of circulating ecdysteroids (Bollenbacher et al., 1981). By day $\mathrm{P}+3$, the two groups diverge with ecdysteroids declining in the diapause-destined animals and rising in those that will continue development. As seen in Figure 3, the patterns of EcR-B1 staining were similar in both short-day- and long-day-reared animals at $48 \mathrm{hr}$ after pupal ecdysis $(n=8)$ but had diverged markedly by $72 \mathrm{hr}(n=8)$. Pupae destined for diapause failed to show the prominent upregulation of EcR-B1 typically seen on fiber 1. Instead, all fibers showed a decline in staining intensity and a decreased number of EcR-B1-labeled nuclei.

The low levels of ecdysteroid present during the first $48 \mathrm{hr}$ after pupal ecdysis are essential for degeneration of the myonuclei throughout the five fibers of muscle DEO1 (Hegstrom and Truman, 1996a). The role of this low level of ecdysteroids on EcR-B1 expression was examined by ligation. Muscles DEO1 of abdomens isolated on day P0 showed no or very low numbers of EcR-B1labeled nuclei when examined $48(n=6)$ and $72(n=6)$ hr later (Fig. 4). A single injection of $10 \mu \mathrm{g}$ of $20 \mathrm{E}$ at $24 \mathrm{hr}$ after ligation increased both EcR-B1 staining intensity and the number of labeled nuclei by $24(n=6)$ and $48(n=6)$ hr after injection (Fig. $4)$. The levels of staining induced by this treatment were higher than those typically seen on days $\mathrm{P}+1$ and $\mathrm{P}+2$, and they included persistent staining of all of the fibers of muscle DEO1. The significance of this result will be considered in Discussion.

The timing of the upregulation of EcR-B1 on fiber 1 of muscle DEO1 on day $\mathrm{P}+3$ suggests a possible response to the surge of ecdysteroids that initiates adult differentiation. This possibility was tested using diapausing pupae that were injected with $20 \mu \mathrm{g}$ of $20 \mathrm{E}$ to initiate adult development. As seen in Figure 5 (bottom, from the following experiment), injection of $20 \mathrm{E}$ was followed $\sim 48 \mathrm{hr}$ later by the appearance of EcR-B1-stained nuclei in the muscle rudiment. As seen during normal development, this upregulation of EcR-B1 was confined to a hot spot on fiber 1 .

\section{Relationship of EcR expression to myogenesis}

The hot spot of EcR-B1 expression on fiber 1 of DEO1 corresponds to the site of muscle regrowth and the location of myogenic nuclei that are involved in this growth (as indicated by their incorporation of BrdU; Hegstrom and Truman, 1996a). Diapausing animals were given an injection of $20 \mathrm{E}$ to initiate adult development and then at 24 or 48 hr later were given an injection of BrdU $6 \mathrm{hr}$ before dissection. The paired muscles DEO1 from these animals were then separated, one set being stained for BrdU incorporation, whereas the other was stained for EcR-B1 expression. By $24 \mathrm{hr}$ after injection of $20 \mathrm{E}$, BrdU incorporation was seen in an occasional myonucleus at the hot spot of fiber 1 $(n=4)$, but no EcR expression was yet evident (Fig. $5 ; n=4)$. By $48 \mathrm{hr}$ after injection of $20 \mathrm{E}$, though, both BrdU incorporation and EcR-B1 expression were prominent in the central region of fiber $1(n=4)$.

Figure 6 compares the size of nuclei incorporating BrdU with those that express EcR-B1. The nuclei that incorporate BrdU were of a uniform size at $\sim 6 \mu \mathrm{m}$ in length. A similar number of nuclei in this size class also expressed EcR-B1 by $48 \mathrm{hr}$ after injection of $20 \mathrm{E}$. In addition to these small nuclei, larger nuclei ranging up to $22 \mu \mathrm{m}$ in length showed EcR-B1 immunoreactivity. These large nuclei seemed to fall into distinct size classes that corresponded to multiples of the small $(6 \mu \mathrm{m})$ nuclei. 


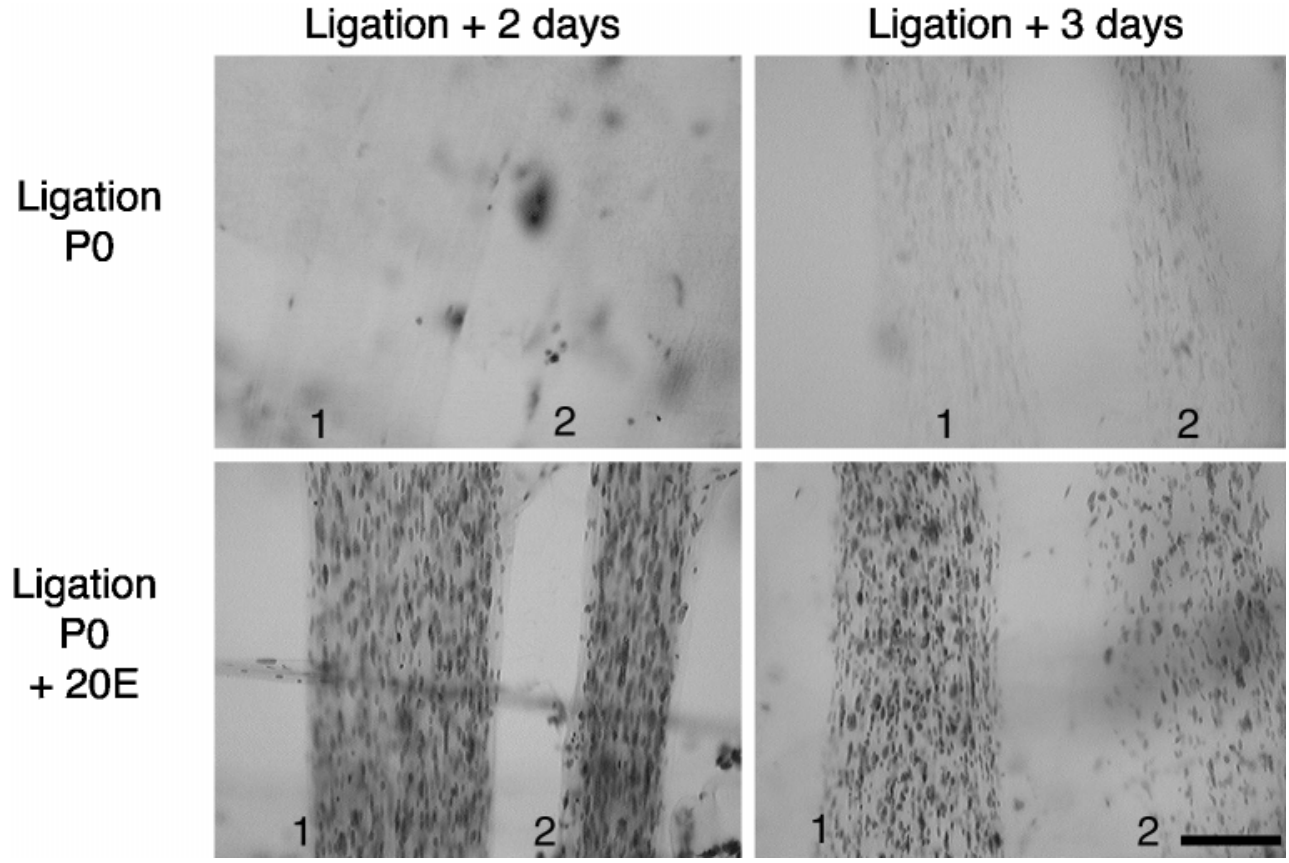

Figure 4. Photomicrographs showing the effects of abdominal isolation and injection with $10 \mu \mathrm{g}$ of $20 \mathrm{E}$ on the expression of EcR-B1 in the first two fibers (numbers) of DEO1. Abdomens were isolated by ligation at day P0. Top, No subsequent treatment. Bottom, Abdomens injected with $20 \mathrm{E}$ at $24 \mathrm{hr}$ after ligation. Fiber 1 is to the left. Scale bar, $100 \mu \mathrm{m}$.
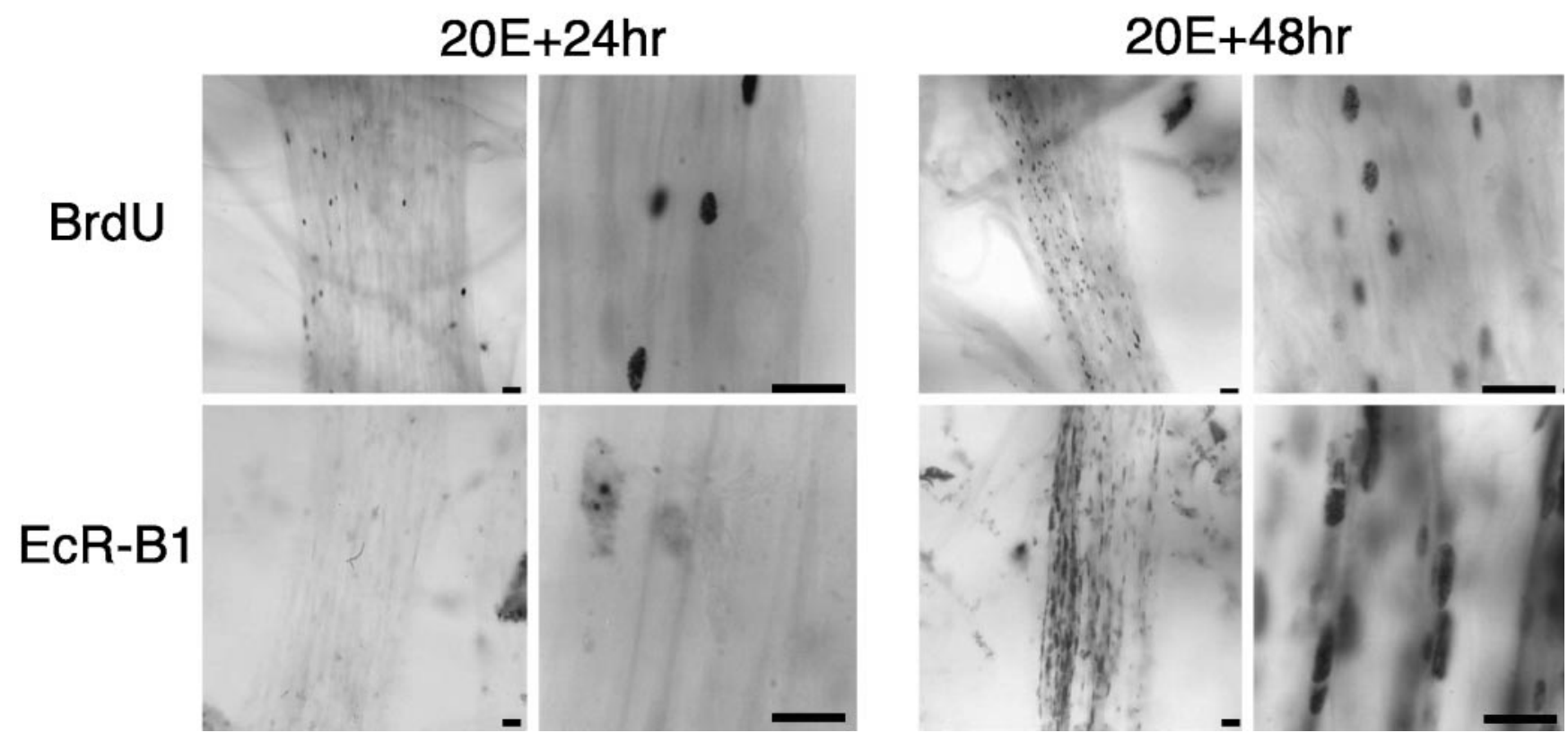

Figure 5. Photomicrographs of fiber 1 of DEO1 showing the time course of response to 20E treatment for BrdU incorporation (top) and EcR-B1 expression (bottom). Diapausing pupae were injected with 20E and examined 24 and $48 \mathrm{hr}$ later. For each time point, EcR-B1 and BrdU staining are of contralateral muscle DEO1s from the same animal. Scale bars, $20 \mu \mathrm{m}$.

\section{Relationship of EcR expression during adult development to the pattern of muscle innervation}

At day $\mathrm{P}+3$, muscle DEO1 showed a distinct heterogeneity in levels of expression of EcR-B1. Myonuclei confined to a hot spot in the central region of fiber 1 corresponding to the site of innervation showed high levels of expression of EcR-B1, whereas the other four fibers showed low to moderate levels of staining, and many fewer nuclei were labeled. To test the dependence of the appearance of EcR-B1 on innervation, we performed unilateral axotomy experiments to denervate one of the paired DEO1 muscles within segment A4 of diapausing pupae. Three days later the animals were injected with $20 \mu \mathrm{g}$ of $20 \mathrm{E}$ to initiate adult development. As seen in Figure $7 A$, the intact side showed the expected increase in EcR-B1, whereas the denervated side failed to show receptor upregulation $(n=4)$. Thus, innervation seems essential for the induction of EcR-B1 expression in the developing muscle.

To determine whether innervation was required to maintain the high levels of EcR-B1, we denervated muscles on day $\mathrm{P}+3$, a time when high levels were present at the hot spot on fiber 1. By $24 \mathrm{hr}$ after denervation, both the number of nuclei expressing EcR-B1 (Fig. 7B) and their intensity of expression were reduced on the denervated, compared with the innervated, side. By $48 \mathrm{hr}$ after denervation, no EcR-B1 staining was evident in denervated 


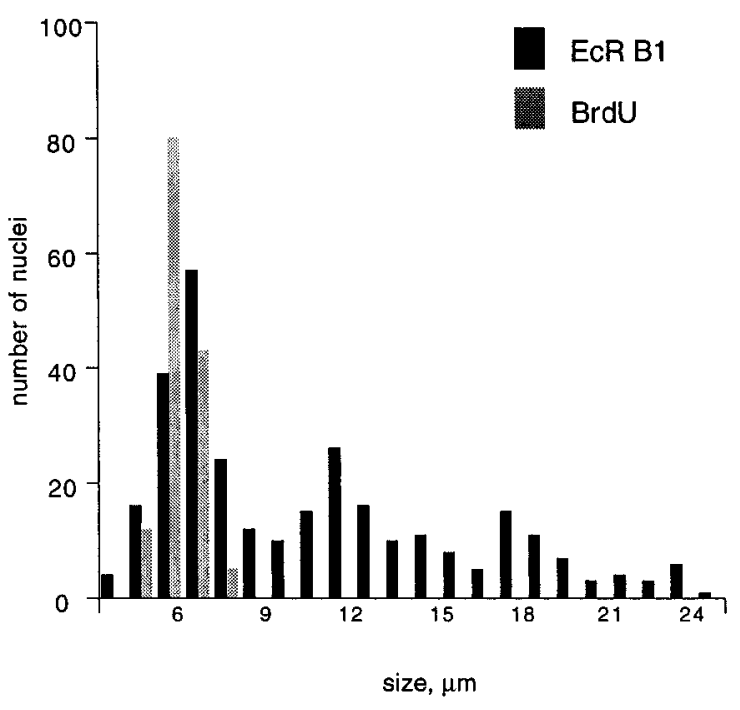

Figure 6. Distribution of the diameter of nuclei in fiber 1 of muscle DEO1 at day P +3 of development. We measured the longest dimension of each nucleus in a $10,000 \mu \mathrm{m}^{2}$ square within the central region of the fiber. Size of nuclei that incorporated BrdU are compared with those expressing EcR-B1.

muscle, whereas normal levels of receptor were retained in the contralateral, innervated muscles (Fig. 7C).

In contrast to the high levels of staining seen in the first fiber at day $\mathrm{P}+3$ after adult differentiation had begun, the low to moderate levels of EcR-B1 seen in all fibers at day $\mathrm{P}+2$ was not dependent on innervation. Long-day-reared pupae were axotomized on day $\mathrm{P} 0$ and subsequently examined at 48 and $72 \mathrm{hr}$ later. At 48 hr there was no significant effect of innervation on the early pattern of EcR staining by muscle DEO1 (Fig. $8 ; n=4$ ). By $72 \mathrm{hr}$ after axotomy, however, the innervated muscle DEO1 showed the upregulation of EcR-B1 expression on fiber $1(n=4)$, whereas the contralateral denervated fiber 1 showed the decreasing staining intensity and fewer EcR-B1-labeled nuclei that was characteristic of the more distal four fibers.

The levels of EcR-A staining on day $\mathrm{P}+2$ were similar to EcR-B1 at that time, because it was expressed equally in all fibers. By contrast, EcR-A levels were not enhanced at the hot spot on fiber 1 by day $\mathrm{P}+3(n=12)$ (Fig. 3$)$. Staining remained at a moderate level in all fibers. Also, in pupae that were unilaterally denervated on day $\mathrm{P} 0$, we saw little difference between denervated and intact sides when examined on day $\mathrm{P}+4(n=6$; data not shown). Thus, EcR-A expression is not dependent on influences from the motoneuron.

\section{Relationship of EcR-B1 levels to degeneration of myonuclei}

There are two steroid-regulated events that occur in the muscle early in the pupal-adult transition (Hegstrom and Truman, 1996a). The degeneration of larval muscle nuclei is dependent on ecdysteroids during the first $24-48 \mathrm{hr}$ after pupal ecdysis, whereas proliferation of myonuclei is caused by the rise of steroids that promotes adult differentiation.

The relationship between EcR-B1 and nuclear degeneration was examined on day $\mathrm{P}+3$ by double labeling nuclei for EcR-B1 and TUNEL. Nuclei early in the process of degrading their DNA invariably co-labeled with TUNEL and with EcR-B1 (Fig. 9). In nuclei where nuclear degeneration was just beginning, EcR-B1 staining and TUNEL-labeled DNA shared a common location in the nucleus. As nuclear degeneration continued, the DNA became tightly condensed, and the EcR-B1 staining was eliminated from those regions and was restricted to regions surrounding the degenerating DNA. Later in the degeneration process, EcR-B1 expression was eliminated from the degenerating nuclei. Measures of intensity of EcR-B1 staining showed that the intensity of staining in nuclei early in the degeneration process was not significantly different from that in nuclei that were not undergoing degeneration $(n=10)$.

\section{DISCUSSION}

\section{EcR expression in DEO1 in relation to early events of adult differentiation}

The receptors for many steroid hormones occur as multiple isoforms that may serve different functions in the cell. In Drosophila, the expression of specific isoforms of EcR are correlated with the developmental fate of the tissue. For example, at pupariation, larval cells prominently express EcR-B1, whereas cells in imaginal disks and imaginal proliferation zones express EcR-A (Talbot et al., 1993). However, the pattern behind the isoform expression is more complex than just larval versus imaginal cells, because larval neurons within the CNS change from one isoform to another as they shift from one pattern of steroid response to another (Truman et al., 1994).

The expression of EcR in muscle DEO1 is dynamic and correlated with specific developmental events. From wandering through the first $48 \mathrm{hr}$ after pupal ecdysis, all fibers of muscle DEO1 expressed EcR uniformly. Comparison of EcR-common, EcR-B1, and EcR-A staining shows that EcR-B1 is prominent during most of the prepupal ecdysteroid peak, but by day $\mathrm{W}+3$ it essentially disappears, leaving only EcR-A. Through this period of the larval-pupal transition, the muscle remains contractile but undergoes a considerable reduction in cross-sectional area. This shifting of EcR isoform expression is correlated with the dismantling of the contractile apparatus of the cell that is triggered by the decline in the prepupal ecdysteroid peak. EcR-A and EcR-B1 expression gradually reappears after pupal ecdysis, and is again evident in all five fibers by day $\mathrm{P}+2$. By day $\mathrm{P}+2$ the fibers lose contractility as cytoplasmic elements degenerate. The myonuclei begin degeneration one day later as a result of the ecdysteroid exposure between day $\mathrm{P}+0$ and $\mathrm{P}+2$ (Hegstrom and Truman 1996a). The mixture of EcR-A and EcR-B1 through this period presumably mediates the action of ecdysteroids in causing this degeneration. The level of EcR expression, however, apparently does not provide the signal for which nuclei will degenerate and which will survive, because all nuclei show a similar pattern of expression through this period. Thus, control of myonuclear degeneration in this muscle is unlike that seen in the Drosophila CNS in which neurons that are doomed to die at the end of metamorphosis uniquely show high levels of EcR-A (Robinow et al., 1993).

In the remodeling leg musculature of Manduca, there is an early aggregation of myoblasts into a muscle anlagen, which then starts active proliferation on day $\mathrm{P}+3$ (Consoulas and Levine, 1997). This proliferation is influenced by both innervation and ecdysteroids, influences that are also seen in vitro when cultured leg myoblasts are supplied with either motoneurons or steroids (Luedeman and Levine, 1996). Abdominal muscles, such as DEO1, are similar, except that these muscles degenerate later than the leg muscles, and the remains of the larval muscles provide a support for adult muscle growth. The roles of innervation and ecdysteroids become evident in the remnants of DEO1 
A

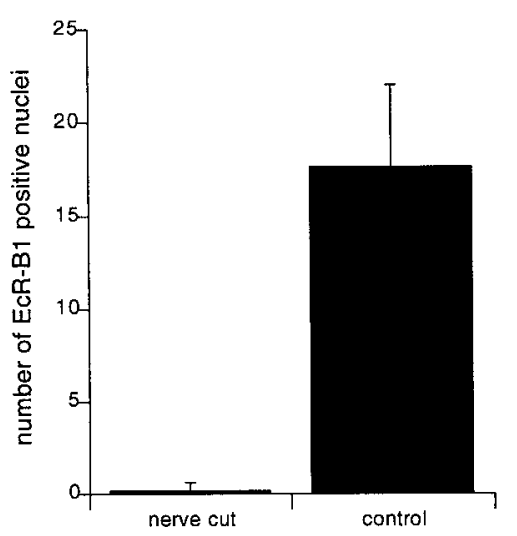

B

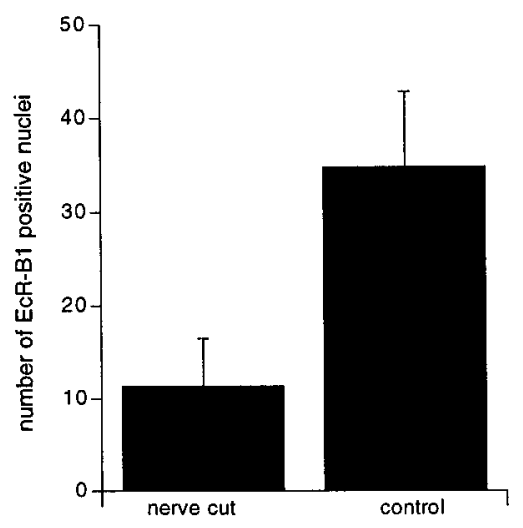

C

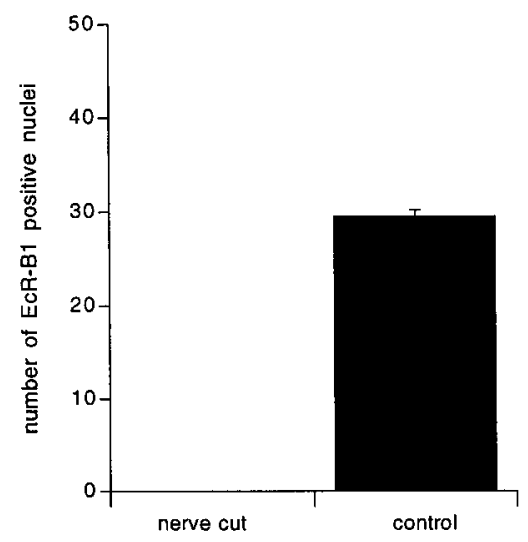

Figure 7. Effect of unilateral axotomy on the number of nuclei expressing EcR-B1. The number of immunostained nuclei within a $10,000 \mu \mathrm{m}^{2}$ square $^{2}$ of the central portion of the intact and denervated fiber 1s were counted. $A$, Axotomy was performed on diapausing pupae; $72 \mathrm{hr}$ later the pupae were injected with 20E to initiate adult development. Muscles were immunostained $48 \mathrm{hr}$ after $20 \mathrm{E}$ injection. $B$, Axotomy was performed on long-day-reared animals at day $\mathrm{P}+3$, and the muscles were immunostained $24 \mathrm{hr}$ later. $C$, Axotomy was performed on long-day-reared animals at day $\mathrm{P}+3$, and the muscles were immunostained $48 \mathrm{hr}$ later. In all cases, the intact sides of the same animals served as controls. All $n=4$.

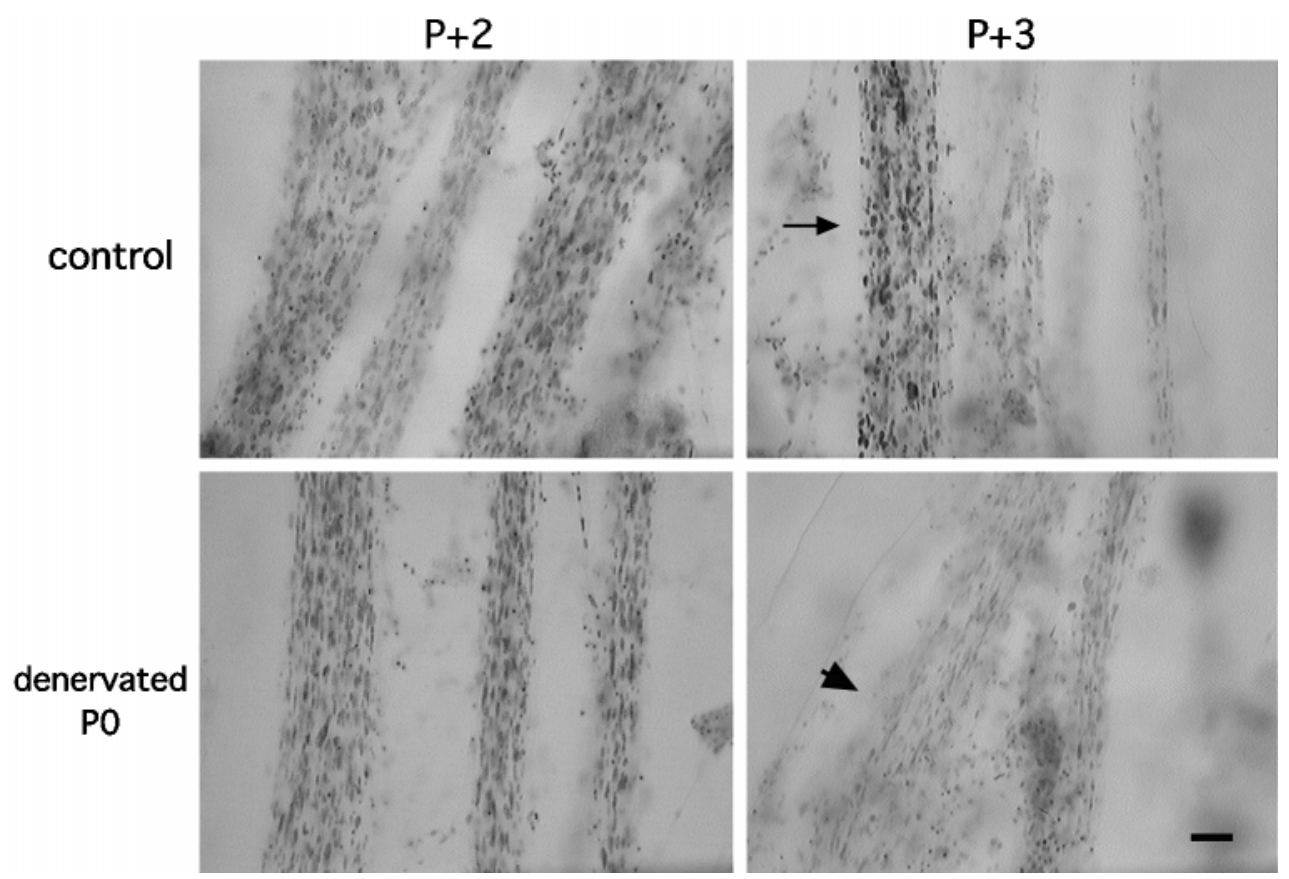

Figure 8. Expression of EcR-B1 in muscle DEO1 after unilateral denervation. One muscle DEO1 was denervated at day $\mathrm{P} 0$, and the state of EcR-B1 expression in the denervated muscle and its contralateral intact homolog was determined 48 and $72 \mathrm{hr}$ later. Top, Control side in which the muscle retained its innervation. Bottom, Denervated DEO1. Arrowhead and arrow indicate fiber 1 . Note that even though these are paired muscles, one member in each pair has been photoreversed, so that fiber 1 is to the left in all cases. Scale bar, $50 \mu \mathrm{m}$. on day $\mathrm{P}+3$ when the five fibers of DEO1 begin to diverge in their developmental responses and in their pattern of EcR expression. The motoneuron retains contact only with the central region of fiber 1. Myonuclei in this region begin to incorporate BrdU as they start replication to form the adult muscle (Hegstrom and Truman, 1996a). Regions of fiber 1 associated with this muscle regrowth consistently show a prominent upregulation of EcR-B1 expression. In a few animals (12\%), we also saw upregulation of EcR-A in this region, but typically EcR-A expression remains uniform across all five fibers. The nuclei that show high EcR-B1 expression range from 6 to $24 \mu \mathrm{m}$ in length. The nuclei in the smallest size class $(6 \mu \mathrm{m})$ also avidly incorporate BrdU. The first signs of nuclear replication are evident slightly before we first detect upregulation of EcR-B1. This discrepancy may be real or it may be attributable to difficulties in discriminating the initial EcR-B1 upregulation using immunocytochemistry.
The larger myonuclei are persistent larval nuclei that may also contribute to the growth of the adult structure. The nature of their contribution is unclear, although in studies of remodeled insect muscle, Nüesch $(1968,1985)$ suggests that the small myogenic nuclei are derived from amitotic divisions of the large myonuclei. In Manduca, we frequently observe large myonuclei that seem to be separating or recently separated into smaller nuclei. We have not been able to determine whether these small nuclei are viable and subsequently undergo replication. The expression of the EcR-B1 isoform does not seem to be necessary for the budding off of large myonuclei, because myonuclei in regions of muscle DEO1 that do not express high levels of EcR-B1 also seem to undergo this fission. Thus, although upregulation of the EcR-B1 isoform to high levels is confined to myonuclei in the regions of muscle regrowth, we do not understand the role of this upregulation in the large nuclei. 

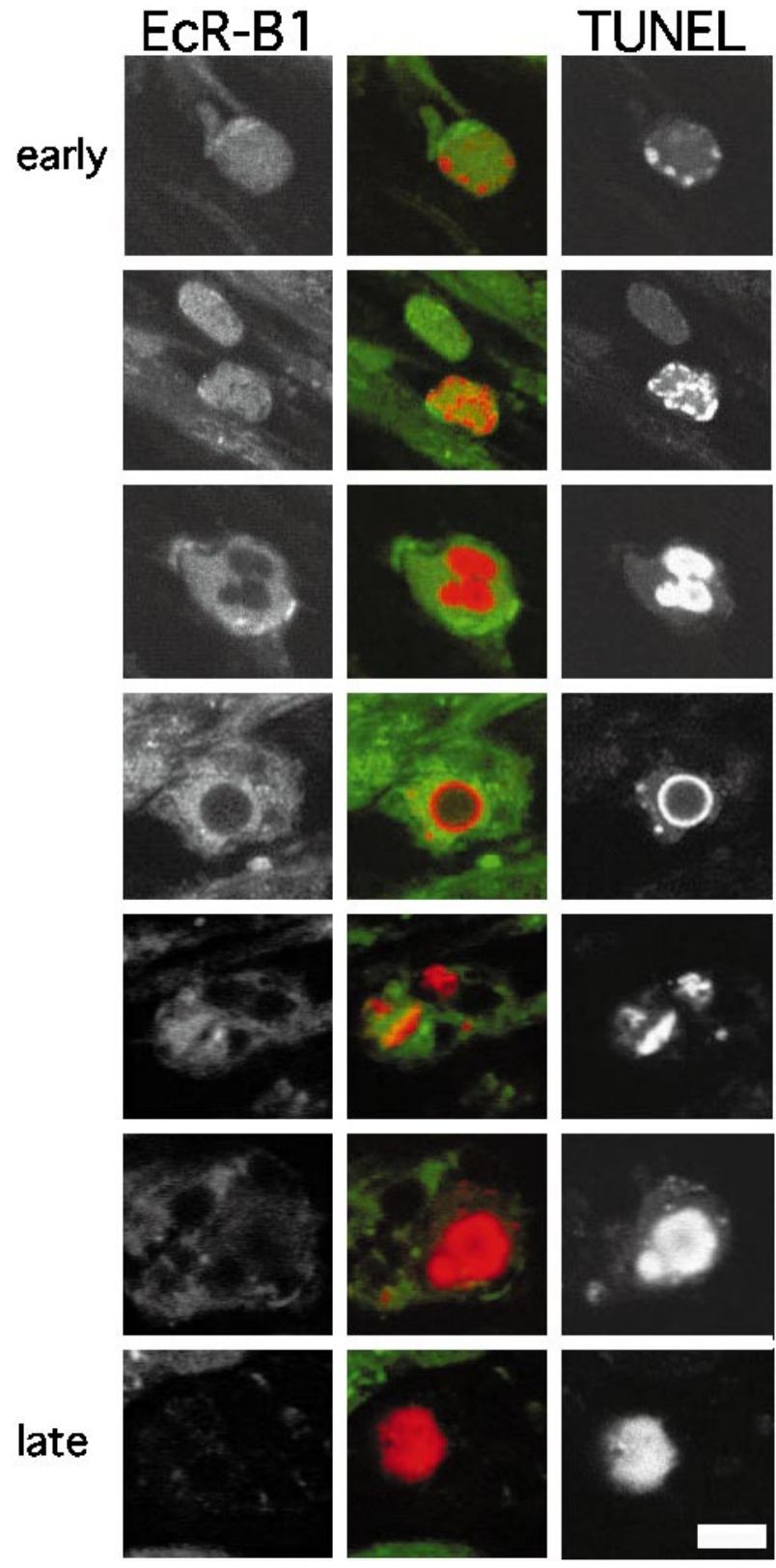

Figure 9. Double label of myonuclei expressing EcR-B1 and undergoing apoptosis. Red, EcR-B1 staining; green, nuclei labeled with the TUNEL method (see Materials and Methods). Presumed progression of myonuclear degeneration is from top to bottom. Nuclei initially label uniformly with EcR-B1, whereas the TUNEL-labeled DNA is confined to clumps within the nuclei. As DNA condenses, EcR-B1 staining is excluded from those regions. Later in the process of degeneration, EcR-B1 staining disappears as the TUNEL labeling spreads throughout the nucleus and the nucleus disintegrates into the cytoplasm. Scale bar, $5 \mu \mathrm{m}$.

\section{Regulation of EcR expression}

A steroid hormone can have both positive and negative effects on the expression of its receptors. Upregulation of EcR by ecdysteroids has been shown to occur in the epidermis of Manduca (Jindra et al., 1996; Hiruma et al., 1997) and is thus similar to what is seen in Drosophila, in which EcR transcripts are induced by $20 \mathrm{E}$ (Karim and Thummel, 1992). In contrast to this upregulation, many steroid hormones have been shown to downregulate the expression of their receptors. For example, estrogen and testosterone can downregulate their receptors (Iwai et al., 1995). In some cases, a steroid hormone can have tissue-specific actions on a specific receptor isoform, as is the case with the type I and type II adrenal steroid receptors (Miller et al., 1993). Also, one receptor isoform may be induced rapidly by a steroid, whereas another in the same tissue may be delayed, resulting in a temporal patterning of receptor expression, as is the case with EcR-B1 and EcR-A in response to 20E in Manduca epidermis (Jindra et al., 1996).

During the larval-pupal transition, the level of EcR-B1 in muscle DEO1 roughly correlates with the prepupal peak of ecdysteroids, suggesting a role of the steroid in upregulating the expression of its receptor. Our experimental tests of the relationship of steroid levels to receptor expression, though, were confined to animals that had entered the pupal stage. When abdomens were isolated at P0 to prevent exposure to the adult peak of endogenous ecdysteroids, levels of EcR-B1 remained low 48-72 hr later. Administration of exogenous $20 \mathrm{E}$ to these isolated abdomens induced EcR-B1 expression. The muscle anlage in these preparations expressed higher levels of EcR-B1 than are normally observed on days $\mathrm{P}+1$ and $\mathrm{P}$ +2 . This high expression is likely attributable to the treatment with $10 \mu \mathrm{g}$ of $20 \mathrm{E}$, a dose of ecdysteroid much higher than that normally found in the Manduca during the first $2 \mathrm{~d}$ after pupal ecdysis (Warren and Gilbert, 1986).

The similarity in the pattern of EcR-B1 expression in muscle of both long-day- and short-day-reared animals during the first $48 \mathrm{hr}$ after pupal ecdysis reflects the common program of development shown by both groups of animals through this time. The groups diverge on day $\mathrm{P}+3$ when diapause-destined animals fail to start the major release of steroid that drives adult differentiation. At this time, the long-day-reared animals express EcR-B1 at high levels in many nuclei of fiber 1, whereas the pupae entering diapause do not. Importantly, injection of $20 \mu \mathrm{g}$ of $20 \mathrm{E}$ into diapausing pupae induces the upregulation of EcR-B1 in fiber 1 within $48 \mathrm{hr}$.

Interactions between cells have also been shown to be important for steroid receptor expression. This has been shown in tissues that interact intimately with one another, such as the contact between a motoneuron and its muscle. In the androgen sensitive levator ani and bulbocavernosus muscles of rats, denervation results in a change in both glucocorticoid receptor (Hughes et al., 1985) and androgen receptor expression (Hughes and Krieg, 1986). Interestingly, in this same system, axotomy of the motoneuron at a critical period of development reduces its expression of androgen receptors, suggesting that the target muscle induces, or is important in inducing, this expression in the neuron (Lubischer and Arnold, 1995).

In Manduca muscle DEO1, when EcR-B1 is upregulated in response to rising ecdysteroid titers on day $\mathrm{P}+3$, this enhanced expression is restricted to the middle region of fiber 1 . During the preceding days, the terminal arbor of the motoneuron has been pruned back from the more distal muscle fibers, so on day $\mathrm{P}+3$ the responding region of the muscle anlagen is the only area that still has contact with a motoneuron (Truman and Reiss, 1995). That innervation is essential for the fiber to respond to ecdysteroids is shown by the results of the denervation experiments. Denervation entirely eliminated the upregulation of EcR-B1 if performed before the upregulation occurred. If performed after the upregulation had commenced, denervation dramatically reduced the expression by $24 \mathrm{hr}$ and eliminated it by $48 \mathrm{hr}$ later. Thus, both exposure to ecdysteroids and local influences from the motoneuron are required for the upregulation and the maintenance of this high level of EcR-B1 expression that is associated with muscle regrowth. By contrast, the pattern of expression of EcR-A is not influenced by the presence or absence of innervation.

Although contact with a motoneuron is necessary for muscle 
regrowth, it is not necessary for the earlier degeneration of the larval myonuclei. Accordingly, innervation is also not required for the earlier phase of moderate EcR-B1 expression (from P0 to P + $2 \mathrm{~d})$ that is associated with this degeneration. The treatment of isolated abdomens with 20E on the day after pupal ecdysis (Fig. 4) shows that precocious treatment with high levels of ecdysteroids can prematurely induce levels of EcR-B1 comparable to those normally seen in the hot spot at later times in development. This high expression, though, is seen in all fibers rather than being confined to fiber 1 . At this early time of steroid treatment, all of the muscle fibers still had functional innervation (Truman and Reiss, 1995). This contact with the motoneuron may then allow the nuclei in the more lateral fibers to respond strongly to the steroid signal.

The nature of the cue(s) from the reorganizing axon terminal arbor is unknown. It is likely diffusible because the effects of the neuron on both EcR-B1 expression and proliferation extend beyond the limits of contact by the reorganizing arbor. Also, we do not know whether the effect of innervation on nuclear proliferation is caused by the upregulation of EcR-B1 or is only correlated with it.

In conclusion, the patterns of the EcR isoforms in muscle DEO1 are influenced by the changing ecdysteroid titers and the local environment experienced by each fiber. Differences in the pattern of receptor isoform expression correlate with differences in the developmental programs initiated by the fibers. Thus, it is the interplay of changing steroid titers, receptor isoform expression, and the local interactions between muscle and motoneuron that orchestrate the developmental programs undertaken by muscle DEO1 during metamorphosis.

\section{REFERENCES}

Antakly T, Raquidan D, O'Donnell D, Katnick L (1990) Regulation of glucocorticoid receptor expression. I. Use of a specific radioimmunoassay and antiserum to a synthetic peptide of the N-terminal domain. Endocrinology 126:1821-1828.

Beato M, Herrlich P, Schultz G (1995) Steroid hormone receptors: many actors in search of a plot. Cell 83:851-857.

Bell RA, Joachim FG (1976) Techniques for rearing laboratory colonies of tobacco hornworms and pink bollworms. Ann Entomol Soc Am 69:365-373.

Bollenbacher WE, Smith SF, Goodman WA, Gilbert, LI (1981) Ecdysteroid titer during larval-pupal-adult development of the tobacco hornworm, Manduca sexta. Gen Comp Endocrinol 44:302-306.

Bradfield JY, Denlinger DL (1980) Diapause development in the tobacco hornworm: a role for ecdysone or juvenile hormone? Gen Comp Endocrinol 41:101-107.

Consoulas C, Levine RB (1997) Accumulation and proliferation of adult leg muscle precursors in Manduca are dependent on innervation. J Neurobiol 32:531-553.

Consoulas C, Anezaki M, Levine RB (1996) Development of adult thoracic leg muscles during metamorphosis of the hawkmoth Manduca sexta. Cell Tissue Res 287:393-412.

Ephrussi B, Beadle GW (1936) A technique of transplantation of Drosophila. Am Nat 70:218-225.

Evans RM (1988) The steroid and thyroid hormone receptor superfamily. Science 240:889-895.

Fujiwara H, Jindra M, Newitt R, Palli SR, Hiruma K, Riddiford LM (1995) Cloning of an ecdysone receptor homolog from Manduca sexta and the developmental profile of its mRNA in wings. Insect Biochem Mol Biol 25:845-856.

Gavrielli Y, Sherman Y, Ben-Sasson SA (1992) Identification of programmed cell death in situ via specific labeling of nuclear DNA fragmentation. J Cell Biol 119:493-501.

Glauser L, Barakat WI (1997) Differential distribution of thyroid hormone receptor isoform in rat dorsal root ganglia and sciatic nerve in vivo and in vitro. J Neuroendocrinol 9:217-227.

Hegstrom CD, Truman JW (1996a) Steroid control of muscle remodeling during metamorphosis in Manduca sexta. J Neurobiol 29:535-550.

Hegstrom CD, Truman JW (1996b) Synapse loss and axon retraction in response to local muscle degeneration. J Neurobiol 31:175-188.
Hiruma K, Bocking D, Lafont R, Riddiford LM (1997) Action of different ecdysteroids on the regulation of mRNA's for the ecdysone receptor, MHR3, dopa decarboxylase, and a larval cuticle protein in the larval epidermis of the tobacco hornworm, Manduca sexta. Gen Comp Endocrinol 107:84-97.

Hughes BJ, Krieg M (1986) Increased glucocorticoid/androgen receptor ratios in denervated striated muscle. J Steroid Biochem Mol Biol 25:695-699.

Hughes BJ, Rybezynska M, Lammel A, Drieg M (1985) Effect of denervation or castration on steroid receptors in rat bulbocavernosus/ levator ani muscles. J Steroid Biochem Mol Biol 23:907-912.

Iwai M, Kanzaki H, Fujimoto M, Kojima K, Hatayama H, Inoue T, Higuchi T, Nakayama H, Mori T, Fujita J (1995) Regulation of sex steroid receptor gene expression by progesterone and testosterone in cultured human endometrial stromal cells. J Clin Endocrinol Metab 80:450-454.

Jindra M, Malone F, Hiruma K, Riddiford LM (1996) Developmental profiles and ecdysteroid regulation of the mRNAs for two ecdysone receptor isoforms in the epidermis and wings of the tobacco hornworm, Manduca sexta. Dev Biol 180:258-272.

Karim FD, Thummel CS (1992) Temporal coordination of regulatory gene expression by the steroid hormone ecdysone. EMBO J 11:4083-4093.

Kawahara A, Baker B, Tata J (1991) Developmental and regional expression of thyroid hormone receptor genes during Xenopus metamorphosis. Development 112:933-943.

Koelle MR, Talbot WS, Seagraves WA, Bender MT, Cherbas P, Hogness DS (1991) The Drosophila EcR gene encodes an ecdysone receptor: a new member of the steroid receptor superfamily. Cell 67:59-77.

Levine RB, Truman JW (1985) Dendritic reorganization of abdominal motoneurons during metamorphosis of the moth, Manduca sexta. J Neurosci 5:2424-2431.

Lubischer J, Arnold A (1995) Axotomy transiently downregulates androgen receptors in motoneurons of the spinal nucleus of the bulbocavernosus. Brain Res 694:61-68.

Luedeman R, Levine RB (1996) Neurons and ecdysteroids promote the proliferation of myogenic cells cultured from the developing adult legs of Manduca sexta. Dev Biol 173:51-68.

Miller AH, Spencer RL, Husain A, Rhee R, McEsen BS, Stein M (1993) Differential expression of type I adrenal steroid receptors in immune tissues is associated with tissue-specific regulation of type II receptors by aldosterone. Endocrinology 133:2133-2140.

Nüesch, H (1968) The role of the nervous system in insect morphogenesis and regeneration. Annu Rev Entomol 13:27-44.

Nüesch, H (1985) Control of muscle development. In: Comparative insect physiology, biochemistry and pharmacology, Vol 2 (Keerkut GA, Gilbert LI, eds), pp 425-452. Oxford: Pergamon.

Robinow, S, Talbot WS, Hogness DS, Truman JW (1993) Programmed cell death in the Drosophila CNS is ecdysone-regulated and coupled with a specific ecdysone receptor isoform. Development 119:1251-1259.

Schmidt TJ, Mayer AS (1994) Autoregulation of corticosteroid receptors. How, when, where and why? Receptor 4:229-257.

Talbot WS, Swyryd EA, Hogness DS (1993) Drosophila tissues with different metamorphic responses to ecdysone express different ecdysone receptor isoforms. Cell 73:1323-1337.

Truman JW, Reiss S (1995) Neuromuscular metamorphosis in the moth Manduca sexta: hormonal regulation of synapse elimination and sprouting. J Neurosci 15:4815-4826.

Truman JW, Talbot WS, Fahrbach SE, Hogness DS (1994) Ecdysone receptor expression in the CNS correlates with stage-specific responses to ecdysteroids during Drosophila and Manduca development. Development 120:219-234.

Warren JT, Gilbert LI (1986) Ecdysone metabolism and distribution during the pupal-adult development of Manduca sexta. Insect Biochem 16:65-82.

Watson AH, Burrows M (1981) Input and output synapses on identified motor neurones of a locust revealed by the intracellular injection of horseradish peroxidase. Cell Tissue Res 215:325-332.

Weeks JC, Roberts WM, Trimble DL (1992) Hormonal regulation and segmental specificity of motoneuron phenotype during metamorphosis of the tobacco hornworm, Manduca sexta. Dev Biol 149:185-196.

Wills KN, Zhang X, Pfahl M (1991) Coordinate expression of functionally distinct thyroid receptor alpha isoforms during neonatal brain development. Mol Endocrinol 5:1109-1119.

Yaoita Y, Brown DD (1990) A correlation of thyroid hormone receptor gene expression with amphibian metamorphosis. Proc Natl Acad Sci USA 87:7090-7094. 